International Journal of Pure and Applied Mathematics

Volume 83 No. 1 2013, 13-23

ISSN: 1311-8080 (printed version); ISSN: 1314-3395 (on-line version)

url: http://www.ijpam.eu

doi: http://dx.doi.org/10.12732/ijpam.v83i1.3

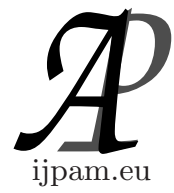

\title{
ON THE STRUCTURE OF RESOLUTIONS OF ALMOST COMPLETE INTERSECTIONS
}

\author{
Alfio Ragusa ${ }^{1}$, Giuseppe Zappalà2 $§$ \\ 1,2 Dipartimento di Matematica e Informatica \\ Università di Catania \\ Viale A. Doria 6, 95125 Catania, ITALY
}

\begin{abstract}
We provide a structure theorem for all almost complete intersection ideals of depth three in any Noetherian local ring. In particular, we find that the minimal generators are the pfaffians of suitable submatrices of an alternating matrix.
\end{abstract}

AMS Subject Classification: 13D40, $13 \mathrm{H} 10$

Key Words: almost complete intersections, Gorenstein rings, pfaffians, Betti numbers

\section{Introduction}

One of the most important and useful tool on studying projective algebraic varieties, in particular for those that are arithmetically Cohen Macaulay, consists on producing a "canonical" (graded) free resolution (possibly minimal) for certain classes of such varieties. Of course, the same question arises in the study of ideals on a Noetherian local ring, in particular on studying perfect ideals. The importance of these results lies, not only in characterizing the fixed class, but

Received: February 10, 2012

(C) 2013 Academic Publications, Ltd. url: www.acadpubl.eu

${ }^{\S}$ Correspondence author 
also in the possibility to calculate some fundamental numerical invariants of the varieties as the degree, the regularity of Castelnuovo-Mumford, the Hilbert function, the graded Betti numbers, the Cohen-Macaulay type and so on. Some of the headlines of the kind of results are, for example, the Koszul resolution for complete intersections (Hilbert's Theorem), the Hilbert-Burch Theorem for perfect ideals of height 2 or for arithmetically Cohen-Macaulay projective schemes of codimension 2, the Buchsbaum-Eisenbud Theorem for Gorenstein ideals of height 3 in Noetherian local rings or for arithmetically Gorenstein projective schemes of codimension 3 . In this work we deal with another important class of varieties, which generalizes from one side the complete intersections and on the other side is closely related to Gorenstein schemes. Namely, we deal with almost complete intersections, precisely, those aCM schemes $X$ of $\mathbb{P}_{k}^{r}$, of codimension $c$ whose defining ideal $I_{X}$ is minimally generated by $c+1$ elements or, from an algebraic point of view, those perfect ideals $I$ of any Noetherian local ring $A$ for which $\nu(I)=\operatorname{depth} I+1$. Some information about almost complete intersection schemes can be found in the J. Migliore book's [Mi]. Indeed, very little is known about almost complete intersection schemes (or ideals), for instance some result for "generic" almost complete intersections is in the article of J. Migliore and R. Miró-Roig $[\mathrm{MM}]$. Clearly, almost complete intersection schemes have the minimal number of generators with respect to the codimension apart from complete intersection schemes. On the other hand, the link with the Gorenstein schemes stems from the simple observation that if we perform a direct complete intersection linkage of a Gorenstein scheme using a complete intersection which contains it, we get a scheme which is just an almost complete intersection. In fact, if $I_{Q} \subset R$ is the defining ideal of an almost complete intersection of codimension $c$ and $I_{Z} \subseteq I_{Q}$ is generated by $c$ minimal generators of $I_{Q}$ which form a regular sequence then $I_{G}:=I_{Z}: I_{Q}$ is the defining ideal of an aG scheme. By liaison theory (see for instance [PS]) we have also $I_{Q}=I_{Z}: I_{G}$. Now, using this fact and the very nice structure theorem for aG schemes of codimension 3 (Buchsbaum-Eisenbud [BE]), in this paper we will give a structure theorem for all almost complete intersections of depth 3 (see Theorem 3.3). S. Seo in Theorem 2.4 of [Se] gives a characterization for almost complete intersections $I_{Q}$ in the special case in which the aG scheme $I_{G}:=I_{Z}: I_{Q}$ is minimally generated by a set of elements where the three of the smallest degrees form a regular sequence. Here we give our structure theorem, in a different setting, for all almost complete intersections. As a simple consequence of our result we obtain a characterization for all almost complete intersection ideals $I_{Q}$, with $\operatorname{depth} I_{Q}=3$. Precisely, they are the ideals for which there exists an alternating matrix $M$ of odd rank $m$, such that three among the submaxiaml pfaffians 
of $M$ say $p_{a}, p_{b}, p_{c}$ form a regular sequence and $I_{Q}$ is minimally generated by $p_{a}, p_{b}, p_{c}, p_{a b c}$, where $p_{a b c}$ is the $(m-3)$-pfaffian obtained by $M$ deleting the lines $a, b, c$ (see Corollary 3.2). The resolution which we produce for the almost complete intersections is not in general minimal, anyway it is very close to be minimal in the sense that we indicate the only possible cancellations which in any case are at most three and, indeed, in many cases the resolution obtained is minimal. This work is part of a screening program that also includes the characterization of the Hilbert function and the graded Betti numbers for all almost complete intersections of codimension 3. Some of these results can be found in [RZ].

\section{Notation and preliminaries}

Let $R$ be a Noetherian local ring. A perfect ideal $I$ of $R$ is called an almost complete intersection ideal if $\nu(I)=\operatorname{depth} I+1$ (here $\nu(I)$ denotes the number of minimal generators of $I$ ). Of course, in case $R$ is Cohen-Macaulay an ideal $I$ is an almost complete intersection when $\nu(I)=h t I+1$, where ht $I$ is its height or codimension. Analogously, let $k$ be an algebraically closed field and $X \subset \mathbb{P}_{k}^{r}$ be a closed subscheme of codimension $c$. Let $I_{X} \subset R=k\left[x_{0}, x_{1}, \ldots, x_{r}\right]$ be the saturated homogeneous ideal defining $X$. Then $X \subset \mathbb{P}_{k}^{r}$ is said an almost complete intersection scheme when its defining ideal $I_{X}$ is perfect and minimally generated by $c+1$ elements. Our first observation is that every almost complete intersecion is directly linked in a complete intersection to an arithmetically Gorenstein $(\mathrm{aG})$ scheme. Indeed, if $I_{Q} \subset R$ is the defining ideal of an almost complete intersection of heigth $c$ and $I_{Z} \subseteq I_{Q}$ is generated by $c$ minimal generators of $I_{Q}$ which form a regular sequence then $I_{G}:=I_{Z}: I_{Q}$ is the defining ideal of an aG scheme. By liaison theory (see [PS] for a complete discussion on this argument) we have also $I_{Q}=I_{Z}: I_{G}$.

Let $R$ be any commutative ring.

If $i \neq j$ are positive integers we set $\langle i, j\rangle$ the following integer

$$
\langle i, j\rangle=\left\{\begin{array}{ll}
i+j+1 & \text { if } i<j \\
i+j & \text { if } i>j
\end{array} .\right.
$$

If $M=\left(a_{i j}\right)$ is an alternating matrix of size $m$ with entries in $R$ we will denote by $M_{\widehat{i j}}$ the alternating matrix obtained from $M$ by deleting both rows and columns $i$ and $j$. With this terminology when $m$ is even one can easily verify that the pfaffian of $M$ (for definitions and basic facts on the pfaffians see for 
instance $[\mathrm{IK}]$ Appendix B) can be computed, for every $i=1, \ldots, m$, by

$$
\operatorname{pf} M=\sum_{j \neq i}(-1)^{\langle i, j\rangle} a_{i j} \text { pf } M_{\widehat{i j}}
$$

Moreover, if $M$ is an alternating matrix of size $m$, with $m$ even, we will denote by $\bar{M}=\left(\bar{a}_{i j}\right)$, where $\bar{a}_{i j}=(-1)^{\langle i, j\rangle}$ pf $M_{\widehat{i j}}$, the pfaffian adjoint of $M$, which is clearly an alternating matrix.

Remark 2.1. If

$$
M=\left(\begin{array}{ccc|c}
0 & a & D \\
-a & 0 & \mid & \\
-- & -- & - & -- \\
-{ }^{t} D & & \mid & C
\end{array}\right)
$$

is an even alternating matrix, by repeating the previous formula for $i=1,2$, one can show that its pfaffian can be computed as

$$
\operatorname{pf} M=a \operatorname{pf} C+\operatorname{pf}\left(D \bar{C}^{t} D\right)
$$

where $\bar{C}$ is the pfaffian adjoint of $C$ as defined above.

Let $\psi: F^{\vee} \rightarrow F$ be an alternating map with $F$ a free $R$-module of rank even $m$. If $B$ is a basis for $F, B^{\vee}$ is the dual basis for $F^{\vee}$ and $M$ is the matrix associated to $\psi$ with respect to such bases, we will denote by $\bar{\psi}: F \rightarrow F^{\vee}$ the map whose associated matrix with respect to the previous bases is the pfaffian adjoint of $M$, i.e. $M(\bar{\psi})=\bar{M}$. Note that $\bar{\psi} \psi=(\operatorname{pf} \psi) \mathrm{id}_{F} \vee$ and $\psi \bar{\psi}=(\mathrm{pf} \psi) \mathrm{id}_{F}$. Moreover we will write $\operatorname{Pf}_{s}(\psi)$ for the ideal generated by the pfaffians of $\psi$ of order $s$.

Furthermore, with the same notation, let us suppose that the rank $m$ is odd and that $p_{1}, \ldots, p_{m}$ are the $m$ submaximal pfaffians of the matrix $M=M(\psi)$ (with respect to fixed bases $B$ and $B^{\vee}$ ). If $p \in J=\left(p_{1}, \ldots, p_{m}\right)$, then there exists an alternating map $\widetilde{\psi}: G^{\vee} \rightarrow G$, where $G$ is a free module of rank $m+2$, such that the alternating matrix $\widetilde{M}=M(\widetilde{\psi})$ (with respect to suitable bases $\widetilde{B}$ and $\widetilde{B}^{\vee}$ ) has the $m+2$ submaximal pfaffians $\left\{p_{1}, \ldots, p_{m}, p, 0\right\}$. Indeed, if $p=a_{1} p_{1}+\ldots+a_{m} p_{m}$, it is enough to take $H=F \oplus R^{2}$ and as $\widetilde{\psi}: H^{\vee} \rightarrow H$, the alternating map which, respect to given bases $\widetilde{B}$ and $\widetilde{B}^{\vee}$, is defined by the 
alternating matrix

$$
\widetilde{M}=\left(\begin{array}{cccc|ccc} 
& & & & 0 & a_{1} \\
& M & & & \vdots & \vdots \\
& & & & 0 & a_{m} \\
-- & -- & -- & -- & -- & -- & -- \\
0 & \cdots & 0 & & 0 & -1 \\
-a_{1} & \cdots & -a_{m} & \mid & 0
\end{array}\right)
$$

To complete the assertion it is enough to compute the submaximal pfaffians of $\widetilde{M}$, using the above formula (1) with respect to the $(m+1)$-th column for all pfaffians except for the $(m+1)$-th pfaffian for which we use formula (1) with respect to the last column. Applying the previous observation we get the following

Lemma 2.2. Let $R$ be a Noetherian local ring and $J$ a Gorenstein ideal of depth 3 in $R$ and $p_{1}, p_{2}, p_{3} \in J$. Then there exists an alternating map $\varphi: H^{\vee} \rightarrow$ $H$ of odd rank $m$ such that $\operatorname{Pf}_{m-1}(\varphi)=J$ and 3 of the submaximal pfaffians of $\varphi$ are exactly $p_{1}, p_{2}, p_{3}$.

Proof. Take any alternating map $\psi: F^{\vee} \rightarrow F$ of rank odd $n$ such that $\operatorname{Pf}_{n-1}(\psi)=J=\left(g_{1}, \ldots g_{n}\right)$, then apply the previous observation to get a new alternating map $\varphi: H^{\vee} \rightarrow H$ of odd rank $m=n+6$ such that $\operatorname{Pf}_{m-1}(\varphi)=$ $J=\left(g_{1}, \ldots g_{n}, p_{1}, p_{2}, p_{3}, 0,0,0\right)$.

We need also the following lemma.

Lemma 2.3. Let $M$ be an alternating matrix of odd size $m$, with entries in a unitary commutative ring $R$. Let $I$ be the ideal generated by the submaximal pfaffians of $M$ and let $\left(q_{1}, \ldots, q_{m}\right)$ be a set of generators of $I$. Then there exists an alternating matrix whose submaximal pfaffians are $u q_{1}, \ldots, u q_{m}$, where $u$ is a unit of $R$.

Proof. Let $\mathbf{p}=\left(p_{1} \ldots p_{m}\right)$ be the vector of the submaximal pfaffians of $M$ and let $\mathbf{q}=\left(q_{1} \ldots q_{m}\right)$. Then $\mathbf{p}=\mathbf{q} A$, where $A$ is an invertible square matrix of size $m$.

Let us consider the alternating matrix $A M^{t} A$. Now, if $N$ is an alternating matrix we write pf $N$ for the vector of the submaximal pfaffians of $N$ and if $A$ is a square matrix we write $\bar{A}$ for the adjoint matrix of $A$. A straightforward computation shows that

$$
\operatorname{pf}\left(A M^{t} A\right)=(\operatorname{pf} M) \bar{A}=\mathbf{p} \bar{A}=(\operatorname{det} A) \mathbf{q} .
$$


Since $A$ is invertible, $\operatorname{det} A$ is a unit, so we are done.

\section{Structure theorem for almost complete intersections}

In the sequel, if $f: M \rightarrow N$ and $g: M \rightarrow P$ are maps of modules we will write $(f, g): M \rightarrow N \oplus P$ for the map defined by $(f, g)(m)=(f(m), g(m))$. Moreover, if $f: M \rightarrow P$ and $g: N \rightarrow P$ we will write $f \mid g: M \oplus N \rightarrow P$ for the map defined by $f \mid g(m, n)=f(m)+g(n)$.

Let $R$ be a Noetherian local commutative ring, $H_{0}$ a free $R$-module of odd rank $m_{0} \geq 5$ and $\varphi_{0}: H_{0}^{\vee} \rightarrow H_{0}$ an alternating map. Let $J:=\operatorname{Pf}_{m_{0}-1}\left(\varphi_{0}\right)$ be the ideal generated by the pfaffians of $\varphi_{0}$ of size $m_{0}-1$ (note that despite the fact that the pfaffians depend on the choice of the bases in $H_{0}, J$ depends only on the map $\left.\varphi_{0}\right)$. Moreover, we denote by pf $\varphi_{0}: H_{0} \rightarrow R$ the map defined by the submaximal pfaffians of $\varphi_{0}$. It is known by Buchsbaum-Eisenbud Theorem (see $[\mathrm{BE}]$ ) that $\operatorname{depth} J \leq 3$ and we suppose here that $\operatorname{depth} J=3$. Take a regular sequence $\left(p_{1}, p_{2}, p_{3}\right)$ in $J$. By Lemma 2.2 there exists an alternating map $\varphi: H^{\vee} \rightarrow H$ of odd rank $m$ such that $\operatorname{Pf}_{m-1}(\varphi)=J$ and 3 of the submaximal pfaffians of $\varphi$ are exactly $p_{1}, p_{2}, p_{3}$. So $J=\left(p_{1}, p_{2}, p_{3}, \ldots, p_{m}\right)$, where the $p_{i}$ 's, for $1 \leq i \leq m$, are the submaximal pfaffians of $\varphi$.

Let $I=\left(p_{1}, p_{2}, p_{3}\right)$ and $\psi: G^{\vee} \rightarrow G$ an alternating map whose pfaffians are exactly $p_{1}, p_{2}, p_{3}$. Then $H=G \oplus F$, where $F$ is a free $R$-module of rank $m-3$, and pf $\varphi=\operatorname{pf} \psi \mid \sigma$, where $\sigma: F \rightarrow R$. Therefore we have the following decomposition: $\varphi=\left(\alpha\left|-\lambda^{\vee}, \lambda\right| \beta\right)$, where $\alpha: G^{\vee} \rightarrow G, \beta: F^{\vee} \rightarrow F$ and $\lambda: G^{\vee} \rightarrow F$. Moreover we denote by $\bar{\beta}: F \rightarrow F^{\vee}$, the alternating map such that $\beta \bar{\beta}=p$ id and $\bar{\beta} \beta=p$ id, where $p$ is the pfaffian of the map $\beta$. One can see that (with respect to suitable bases) the matrix associated to $\bar{\beta}$ is the pfaffian adjoint of the matrix of $\beta$.

Note that pf $\psi^{\vee}=-(\operatorname{pf} \psi)$; moreover we will set the pfaffian of an empty matrix equal to 1 .

Theorem 3.1. With the above notation

$$
0 \longrightarrow F^{\vee} \stackrel{\left(\lambda^{\vee},-\beta\right)}{\longrightarrow} G \oplus F \stackrel{\left(p\left|\lambda^{\vee} \bar{\beta},-\operatorname{pf} \psi\right|-\sigma\right)}{\longrightarrow} G \oplus R \stackrel{\operatorname{pf} \psi \mid p}{\longrightarrow} R
$$

is a free resolution of an almost complete intersection algebra $R / Q$.

Proof. We start by proving that the canonical surjection $R / I \rightarrow R / J$ can be lifted to a map between their resolutions in the following way: 


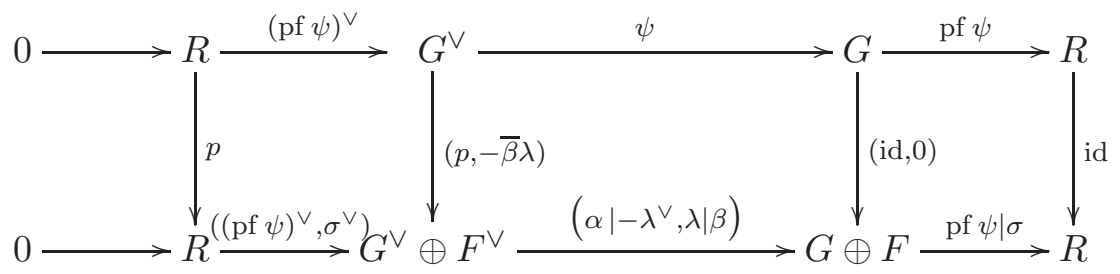

Indeed, since

$$
\begin{gathered}
0=(\lambda \mid \beta)\left((\operatorname{pf} \psi)^{\vee}, \sigma^{\vee}\right)=\lambda(\operatorname{pf} \psi)^{\vee}+\beta \sigma^{\vee} \Rightarrow \\
\Rightarrow \bar{\beta} \lambda(\operatorname{pf} \psi)^{\vee}+\bar{\beta} \beta \sigma^{\vee}=0 \Rightarrow \bar{\beta} \lambda(\operatorname{pf} \psi)^{\vee}+p \sigma^{\vee}=0
\end{gathered}
$$

we have

$$
(p,-\bar{\beta} \lambda)(\operatorname{pf} \psi)^{\vee}=\left(p(\operatorname{pf} \psi)^{\vee},-\bar{\beta} \lambda(\operatorname{pf} \psi)^{\vee}\right)=\left(p(\operatorname{pf} \psi)^{\vee}, p \sigma^{\vee}\right)=p\left((\operatorname{pf} \psi)^{\vee}, \sigma^{\vee}\right)
$$

Now Remark 2.1 will imply, in our notation, that $\alpha p+\lambda^{\vee} \bar{\beta} \lambda=\psi$. Therefore we have

$$
\begin{aligned}
& \left(\alpha\left|-\lambda^{\vee}, \lambda\right| \beta\right)(p,-\bar{\beta} \lambda)= \\
& \quad=\left(\alpha p+\lambda^{\vee} \bar{\beta} \lambda, \lambda p-\beta \bar{\beta} \lambda\right)=(\psi, \lambda p-p \lambda)=(\psi, 0)=(\mathrm{id}, 0) \psi .
\end{aligned}
$$

Then we set $\mathbb{F}_{\bullet}^{I}$ and $\mathbb{F}_{\bullet}^{J}$ the above resolutions of $R / I$ and $R / J$ and $\tau$ : $\mathbb{F}_{\bullet}^{I} \rightarrow \mathbb{F}_{\bullet}^{J}$ the above complex map. Thus, if we set $Q:=I: J$, we see that a free resolution of $R / Q$ is given by the mapping cone of the map $\tau^{\vee}:\left(\mathbb{F}_{\bullet}^{J}\right)^{\vee} \rightarrow\left(\mathbb{F}_{\bullet}^{I}\right)^{\vee}$. If we make cancellations where we have identities map we get the required resolution of the almost complete intersection $R / Q$.

As a simple consequence of the previous result we have

Corollary 3.2. Let $R$ be a Noetherian local ring and let $M$ be an alternating matrix of odd rank $m$, whose entries are in $R$. Let $p_{1}, \ldots, p_{m}$ be the submaximal pfaffians of $M$ and $p_{a b c}$ the pfaffian of order $m-3$ obtained by $M$ by deleting the rows and columns $a, b, c$. If $\left(p_{a}, p_{b}, p_{c}\right)$ is a regular sequence, then

$$
\left(p_{a}, p_{b}, p_{c}\right):\left(p_{1}, \ldots, p_{m}\right)=\left(p_{a}, p_{b}, p_{c}, p_{a b c}\right)
$$

Vice versa if $Q$ is an almost complete intersection ideal of $R$ then there exists an alternating matrix $M$ of odd rank $m$, such that three among the submaxiaml pfaffians of $M$ say $p_{a}, p_{b}, p_{c}$ form a regular sequence and $Q=\left(p_{a}, p_{b}, p_{c}, p_{a b c}\right)$, where $p_{a b c}$ is the $(m-3)$-pfaffian obtained by $M$ deleting the lines $a, b, c$. 
Proof. Since $I:=\left(p_{a}, p_{b}, p_{c}\right)$ is a complete intersection ideal and $J:=$ $\operatorname{Pf}_{m-1}(M)$ is a Gorenstein ideal, $I: J$ is an almost complete intersection ideal. By Theorem 3.1 follows that the generators of $I: J$ are those mentioned in the statement.

Let $I \subset Q$ a complete intersection ideal of depth 3. Then $J:=I: Q$ is a Gorenstein ideal, so Lemma 2.2 will complete the proof.

The Theorem 3.1 gives a way to construct almost complete intersection algebras but the very interesting thing is that every almost complete intersection can be constructed in this way.

Theorem 3.3. Let $R$ be a Noetherian local ring and $Q \subset R$ be a perfect ideal of depth 3 of an almost complete intersection. Then there exists an alternating map $\psi: H^{\vee} \rightarrow H$, where $H$ is a free $R$-module of odd rank with im pf $\psi$ of depth 3, such that, with the above notation,

$$
0 \longrightarrow F^{\vee} \stackrel{\left(\lambda^{\vee},-\beta\right)}{\longrightarrow} G \oplus F \stackrel{\left(p\left|\lambda^{\vee} \bar{\beta},-\operatorname{pf} \psi\right|-\sigma\right)}{\longrightarrow} G \oplus R \stackrel{\operatorname{pf} \psi \mid p}{\longrightarrow} R
$$

is a free resolution of $R / Q$.

Proof. Since $Q$ is an almost complete intersection ideal we have that $Q=$ $\left(p_{0}, p_{1}, p_{2}, p_{3}\right)$ and $\operatorname{since} \operatorname{depth} Q=3$ we can suppose that $I:=\left(p_{1}, p_{2}, p_{3}\right)$ is generated by a regular sequence. Let $J:=I: Q$. Then $J$ is a Gorenstein ideal of depth 3. By Lemma 2.2, there exists an alternating map $\varphi: H^{\vee} \rightarrow H$, such that the submaximal pfaffians of $\varphi$ are exactly $p_{1}, p_{2}, p_{3}$ and the other generators of $J$. By Theorem 3.1 the above resolution gives a resolution of $R / Q$.

Now we would like to give a graded version of the previous result.

Let $\mathbb{P}_{k}^{r}$ be the projective space with $r \geq 3$ and $R=k\left[x_{0}, x_{1}, \ldots, x_{r}\right]$ the standard graded coordinate ring of $\mathbb{P}_{k}^{r}$.

Theorem 3.4. Let $Q \subset \mathbb{P}_{k}^{r}$ be an almost complete intersection scheme of codimension 3 and $I_{Q} \subset R$ be its defining ideal. Then $R / I_{Q}$ admits a graded free resolution of the following type

$$
0 \rightarrow K^{\vee}(-d) \rightarrow G\left(-d_{0}\right) \oplus K \rightarrow G \oplus R\left(-d_{0}\right) \rightarrow R
$$

where $d_{0}$ is a positive integer, $G=\oplus_{i=1}^{3} R\left(-d_{i}\right), d=d_{o}+d_{1}+d_{2}+d_{3}, K=$ $\oplus_{i=4}^{m} R\left(-e_{i}\right), m \geq 5$ an odd integer and $d_{1}, d_{2}, d_{3}, e_{4}-d_{0}, \ldots, e_{m}-d_{0}$ are the degrees of all submaximal pfaffians of a suitable alternating matrix of size $m$. 
Proof. Let $Z \subset \mathbb{P}_{k}^{r}$ be a complete intersection generated by 3 minimal generators of $I_{Q}$, of degrees $d_{1}, d_{2}, d_{3}$. Let $I_{\Gamma}:=I_{Z}: I_{Q} . I_{\Gamma}$ is the saturated homogeneous ideal of an aG scheme $\Gamma$ directly linked to $Q$ in $Z$. As in Theorem 3.1 we have the following diagram

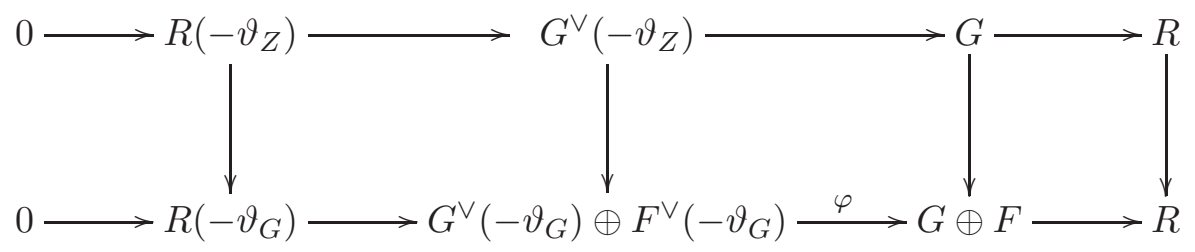

where the first row is a minimal graded resolution of $R / I_{Z}$ and the second row is the graded resolution of $R / I_{\Gamma}$, obtained by the alternating map $\varphi$ as in Lemma 2.2. So we have that $G=\oplus_{i=1}^{3} R\left(-d_{i}\right)$ and $F=\oplus_{i=4}^{m} R\left(-d_{i}\right)$. Dualizing and shifting by $-\vartheta_{Z}$ this diagram we get

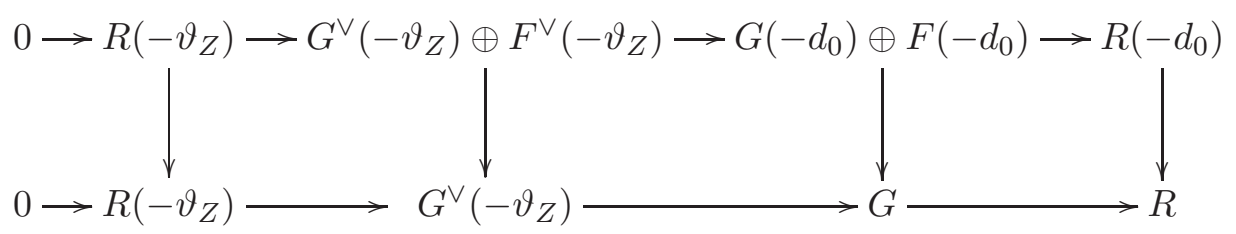

where $d_{0}:=\vartheta_{Z}-\vartheta_{G}$. Taking the mapping cone and after the trivial cancellations we obtain the following resolution of $R / I_{Q}$

$$
0 \rightarrow F^{\vee}\left(-\vartheta_{Z}\right) \rightarrow G\left(-d_{0}\right) \oplus F\left(-d_{0}\right) \rightarrow G \oplus R\left(-d_{0}\right) \rightarrow R .
$$

Now if we set $K:=F\left(-d_{0}\right)$ and $d:=2 \vartheta_{Z}-\vartheta_{G}=d_{0}+d_{1}+d_{2}+d_{3}$ we get

$$
0 \rightarrow K^{\vee}(-d) \rightarrow G\left(-d_{0}\right) \oplus K \rightarrow G \oplus R\left(-d_{0}\right) \rightarrow R,
$$

which is the required resolution.

Corollary 3.5. Let $Q \subset \mathbb{P}_{k}^{r}$ be an almost complete intersection scheme of codimension 3 and $I_{Q} \subset R$ be its defining ideal, minimally generated in degrees $d_{0} \leq d_{1} \leq d_{2} \leq d_{3}$. Then $R / I_{Q}$ admits a graded free resolution of the type

$$
0 \rightarrow K^{\vee}(-d) \rightarrow G\left(-d_{0}\right) \oplus K \rightarrow G \oplus R\left(-d_{0}\right) \rightarrow R,
$$

where $G=\oplus_{i=1}^{3} R\left(-d_{i}\right)$ and $d=d_{0}+d_{1}+d_{2}+d_{3}$.

Proof. Since we can find a regular sequence of minimal generators in $I_{Q}$ of type $d_{1}, d_{2}, d_{3}$, the conclusion follows by repeating the argument of Theorem 3.4 using such a regular sequence. 
Although (2) is not in general a minimal resolution, it is very close to be minimal, as we will see in the next proposition.

Proposition 3.6. Let $Q \subset \mathbb{P}_{k}^{r}$ be an almost complete intersection scheme of codimension 3 and $I_{Q} \subset R$ be its defining ideal, minimally generated in degrees $d_{0} \leq d_{1} \leq d_{2} \leq d_{3}$. Then $R / I_{Q}$ admits a graded free resolution of the type

$$
0 \rightarrow K^{\vee}(-d) \stackrel{\left(\lambda^{\vee},-\beta\right)}{\longrightarrow} \bigoplus_{i=1}^{3} R\left(-d_{0}-d_{i}\right) \oplus K \stackrel{\chi}{\longrightarrow} \bigoplus_{i=1}^{3} R\left(-d_{i}\right) \oplus R\left(-d_{0}\right) \rightarrow R,
$$

where the only possible units can appear in tha matrix associated to the map $\lambda^{\vee}$. In particular we can get from it the minimal free resolution of $I_{Q}$ with at most three cancellations.

Proof. Since $I_{Q}$ is minimally generated by 4 elements a matrix associated to $\chi$ cannot contain units. Then we perform the construction of Lemma 2.2 starting from a minimal free resolution of the linked Gorenstein ideal defined by an alternating matrix $M$. Now we point out that $\beta$ admits as associated matrix a submatrix of $M$, therefore such a matrix has no units.

Example 3.7. Let $Q \subset \mathbb{P}_{k}^{3}$ be the 0-dimensional almost complete intersection linked to 5 general points $(\Gamma)$ in a complete intersection $Z$ of type $(2,2,8)$. Then we consider the following graded resolutions

$$
0 \rightarrow R(-12) \rightarrow R(-4) \oplus R(-10)^{2} \rightarrow R(-2)^{2} \oplus R(-8) \rightarrow R \rightarrow R / I_{Z} \rightarrow 0
$$

and

$$
\begin{aligned}
0 \rightarrow R(-5) \rightarrow R(-8) \oplus R(-3)^{5} \oplus R(3) \rightarrow R(-8) \oplus R(-2)^{5} \oplus R(3) \rightarrow \\
\rightarrow R \rightarrow R / I_{\Gamma} \rightarrow 0
\end{aligned}
$$

where the last one is the pfaffian resolution of $R / I_{\Gamma}$ got by the minimal one by adding the term $R(-8) \oplus R(3)$ to the second and to the third module of the complex. Such a resolution can be built as illustrated in Lemma 2.2 and the pfaffians of its alternating central map are the five minimal generators of $I_{\Gamma}$ of degree 2, the form of $I_{\Gamma}$ of degree 8 used to perform the linkage and the null form. So we have $G=R(-2)^{2} \oplus R(-8), K=R(-9)^{3} \oplus R(-4), d_{0}=7$ and $d=19$. Consequently we get the following resolution of $R / I_{Q}$

$$
0 \rightarrow R(-15) \oplus R(-10)^{3} \rightarrow\left[R(-9)^{2} \oplus R(-15)\right] \oplus\left[R(-9)^{3} \oplus R(-4)\right] \rightarrow
$$




$$
\rightarrow\left[R(-8) \oplus R(-2)^{2}\right] \oplus R(-7) \rightarrow R \rightarrow R / I_{Q} \rightarrow 0 .
$$

Note that to obtain the minimal graded resolution of $R / I_{Q}$ it is enough to deleting from it the term $R(-15)$.

\section{References}

[BE] D.A. Buchsbaum, D. Eisenbud, Algebra structures for finite free resolutions, and some structure theorems for ideals of codimension 3, Amer. J. Math., 99, No. 1 (1977), 447-485, doi: 10.2307/2373926

[IK] A. Iarrobino, V. Kanev, Power Sums, Gorenstein Algebras, and Determinantal Loci, LNM 1721, Springer-Verlag (1999).

[Mi] J. Migliore, Introduction to liaison theory and deficency modules, Progress in Math., 165, Birkhauser Boston (1998).

[MM] J. Migliore, R. Miró-Roig, On the minimal free resolution of $n+1$ general forms, Trans. Am. MAth. Soc., 355, No. 1 (2003), 1-36.

[PS] C. Peskine, L. Szpiro, Liaison des variétés algébriques. I, Inv. Math., 26 (1974), 271-302.

[RZ] A. Ragusa, G. Zappalà, Characterization of the graded Betti numbers for almost complete intersections, Communications in Algerba, To Appear.

[Se] S. Seo, Almost complete intersections, J. of Algebra, 320 (2008), 25942609, doi: 10.1016/j.jalgebra.2008.04.019. 
